\title{
Complete genome sequence of Clostridium perfringens CBA7123 isolated from a faecal sample from Korea
}

\author{
Yeon Bee Kim', Joon Yong Kim', Hye Seon Song' ', Changsu Lee', Joseph Kwon², Jisu Kang 3,4 Jin-Kyu Rhee5,
} Myeong Seon Jeong ${ }^{6}$, Young-Do Nam ${ }^{3,4 t}$ and Seong Woon Roh ${ }^{1 *+}$

\begin{abstract}
Background: Clostridium perfringens is an opportunistic human pathogen that causes necrotic enteritis, mild diarrhea, clostridial myonecrosis or gas gangrene, sepsis, etc. In this study, we aim to determine the pathogenesis of this bacterium at the genomic level. The genome of strain CBA7123 was sequenced, and a comparative genomic analysis between strain CBA7123 and four other related C. perfringens strains was performed.
\end{abstract}

Results: The genome of strain CBA7123 consisted of one circular chromosome and one plasmid that were 3,088,370 and 46,640 bp long with 28.5 and $27.1 \mathrm{~mol} \% \mathrm{G}+\mathrm{C}$ content, respectively. The genomic DNA was predicted to contain 2798 open reading frames (ORFs), 10 rRNA genes, and 94 tRNA genes. The genomic comparison analysis between the five strains revealed the distinctive virulence properties of strain CBA7123 by highlighting certain strain-specific genes.

Conclusions: In this study, the C. perfringens CBA7123 genome was sequenced and compared with other C. perfringens genomes. Among the various genes sequenced, the detection of antimicrobial resistance genes and those encoding various virulence factors may extend the understanding of the pathogenesis of C. perfringens strains.

Keywords: Clostridium perfringens, Complete genome sequence, Comparative genomic analysis, Pathogenesis, Antimicrobial resistant, Virulence factor

\section{Background}

Clostridium perfringens is a Gram-positive, spore-forming, strict anaerobic, rod-shaped bacterium belonging to the phylum Firmicutes [1-3]. This bacterium inhabits diverse environments such as soil, sewage, and animal intestines [4]. Although C. perfringens does not invade healthy cells, it acts as a pathogen by producing various enzymes and toxins and is also considered a common cause of food poisoning worldwide [5]. C. perfringens toxins, mainly comprising $\alpha, \beta, \varepsilon$, and ı extracellular toxins, are indicators for classifying its strains as A to E toxinotypes [6-8]. The type A of C. perfringens is the most commonly found toxinotype that possesses only

\footnotetext{
*Correspondence: swroh@wikim.re.kr

${ }^{\dagger}$ Young-Do Nam and Seong Woon Roh contributed equally to this work

${ }^{1}$ Microbiology and Functionality Research Group, World Institute

of Kimchi, Gwangju 61755, Republic of Korea

Full list of author information is available at the end of the article
}

$\alpha$ toxin-encoding gene, $p l c$. Strains with $\alpha$-toxin genes can cause necrotic enteritis, mild diarrhea, clostridial myonecrosis or gas gangrene, sepsis, and food poisoning in humans as well as various enterotoxemic diseases in livestock $[7,9,10]$. In this study, we aim to elucidate the pathogenesis of this species through its genome, specifically the virulence-related genes. The genome of $C$. perfringens CBA7123 was completely sequenced and analyzed using bioinformatics. Additionally, genomic data that were compared between strain CBA7123 and four other $C$. perfringens strains would illustrate the virulence mechanisms of these bacteria.

\section{Methods \\ Isolation and DNA extraction of C. perfringens CBA7123 \\ Clostridium perfringens CBA7123 (=KCCM 43242) was isolated from the feces of a 73-year-old man, and a pure culture was obtained using serial dilution. The colony}


was cultured anaerobically in ATCC medium no. 2840, modified Eggerth-Gagnon medium (10 g peptone, $4 \mathrm{~g}$ $\mathrm{Na}_{2} \mathrm{HPO}_{4} \cdot 2 \mathrm{H}_{2} \mathrm{O}, 2 \mathrm{~g}$ porcine gastric mucin, $50 \mathrm{ml}$ sheep blood, and $15 \mathrm{~g}$ agar per liter) at $37^{\circ} \mathrm{C}$ for $24 \mathrm{~h}$. The total genomic DNA of strain CBA7123 was extracted using QuickGene DNA tissue kit S (Kurabo, Japan) and the G-spin total DNA extraction kit (iNtRON Biotechnology, Korea). The purity, quality, and quantity of genomic DNA were measured using Agilent 2100 Bioanalyzer (Agilent Technologies, USA) as described the manufacturer's instruction.

\section{Library preparation and genome sequencing}

Detailed genome sequencing was performed as described previously [11]. Briefly, the genomic DNA of strain CBA7123 was sheared according to the PacBio 20-kb Template Preparation using BluePippin Size-Selection System protocol, and SMRTbell library was prepared using P6-C4 chemistry (Pacific Biosciences, USA). The sequences were obtained using PacBio RS II system (Pacific Biosciences) as following an instruction of the manufacturer. The 150,292 reads were generated with 7090 bp of average read length by the PacBio RS II system from one SMRT cell.

\section{Genome assembly and annotation}

De novo assembly of the genome sequence was performed using Hierarchical Genome Assembly Process (HGAP) version 2 software, with default parameters supported by PacBio SMRT Analysis ver. 2.3.0 [12]. rRNA and tRNA of the assembled sequence were identified using RNAmmer 1.2 and tRNAscan-SE 1.21, respectively. Genes were predicted using Glimmer3 of Rapid Annotation using Subsystem Technology (RAST) server (http://rast.nmpdr.org), and functional gene annotations were performed using the SEED, Clusters of Orthologous Groups (COG, http://www.ncbi.nlm.nih.gov/COG), and Kyoto Encyclopedia of Genes and Genomes (KEGG, http://www.genome.jp/kegg/) databases. PathogenFinder 1.1 [13] and ResFinder 2.1 [14] were used to estimate the pathogenicity and antimicrobial resistance genes, respectively. The virulence factors were searched using the Basic Local Alignment Search Tool (BLAST) in the virulence factors of pathogenic bacteria database [15] with default parameters and predicted with zero e-value.

\section{Comparative genomic analysis}

The $C$. perfringens strains for comparative genomic analysis were selected using Microbial Nucleotide BLAST in the NCBI complete genome database. The four strains with BLAST total scores over $5.5 \mathrm{e}+06$ were selected: strains FORC 003, JP55, FORC 025, and JP838. To compare the genomic structures between strain CBA7123 and these four strains, the progressive alignment algorithm in MAUVE multiple genome alignment software 2.4.0 was used [16]. The OrthoANI algorithm was used to analyze genomic relatedness between strain CBA7123 and other C. perfringens species. OrthoANI percentages were calculated and a phylogenetic tree was constructed, as described by Lee et al. [17]. Orthologs between strain CBA7123 and the reference strains were predicted and mapped using the reciprocal best hit method in UBLAST [18]. Pan-genome orthologous groups (POGs) were estimated using the EzBioCloud Comparative Genomics Database (http:// cg.ezbiocloud.net/) [19], and their presence was calculated using the Jaccard coefficient. UPGMA clustering was then used to show clustering between strain CBA7123 and the reference strains as a dendrogram, based on the presence or absence of gene content. The intersections and differences of POG sets of the different strains were visualized as a Venn diagram, using the jvenn program [20].

\section{Quality assurance}

The single colony of strain CBA7123 was transferred more than three times to obtain a pure culture and confirmed using a variable pressure field emission scanning electron microscope (Chuncheon Center, Korea Basic Science Institute, Korea) (Additional file 1: Figure S1) and a PCR-sequencing approach targeting 16S rRNA gene as described by Min et al. [21].

\section{Results and discussion \\ General features}

The genome of strain CBA7123 comprised two circular contigs that were $3,088,370$ and 46,640 bp long with 28.5 and $27.1 \mathrm{~mol} \% \mathrm{G}+\mathrm{C}$ content, respectively. Each contig matched to a chromosome and plasmid by the BLAST analysis with GenBank genome database. 2798 open reading frames (ORFs), 10 rRNA (10 5S rRNA, 10 16S rRNA, 10 23S rRNA) operons, and 94 tRNA genes were present. Among the various COG categories, G (carbohydrate transport and metabolism; 205 ORFs), E (amino acid transport and metabolism; 168 ORFs), J (translation, ribosomal structure, and biogenesis; 163 ORFs), K (transcription; 149 ORFs), and L (replication, recombination, and repair; 147 ORFs) made up the largest proportion ( $\geq 6 \%$ of the total COGs classifications). Detailed genome features are summarized in Table 1, and numbers of COG functional categories are shown in Table 2.

\section{Comparative genomic analysis of strain CBA7123 with other $C$. perfringens strains}

In the comparison of genomic structures between strain CBA7123 and strains FORC 003, JP55, FORC 025, and JP838, locally collinear blocks indicated high 
Table 1 General features of the Clostridium perfringens CBA7123 genome

\begin{tabular}{ll}
\hline Property & Term \\
\hline Finishing quality & Complete \\
Libraries used & SMRTbell library \\
Sequencing platforms & PacBio_20K \\
Assemblers & PacBio SMRT analysis 2.3.0 \\
Pre-filtered reads & 150,292 \\
Post-filtered reads & 77,985 \\
Average genome coverage & $282.57 X$ \\
Genome size (bp) & $3,135,010$ \\
DNA G+C content & 28.5 \\
Total ORFs & 2798 \\
rRNA operons & 10 \\
tRNA genes & 94 \\
\hline
\end{tabular}

Table 2 Number of general COG-associated functional genes

\begin{tabular}{lrrl}
\hline Code & Value & \% age & Description \\
\hline J & 163 & 6.65 & Translation, ribosomal structure and biogenesis \\
K & 149 & 6.08 & Transcription \\
L & 147 & 6.00 & Replication, recombination and repair \\
D & 27 & 1.10 & Cell cycle control, cell division, chromosome \\
& & & partitioning \\
O & 71 & 2.90 & Posttranslational modification, protein turnover, \\
& & & chaperones \\
M & 139 & 5.67 & Cell wall/membrane/envelope biogenesis \\
N & 4 & 0.16 & Cell motility \\
P & 125 & 2.10 & Inorganic ion transport and metabolism \\
T & 96 & 3.92 & Signal transduction mechanisms \\
C & 137 & 5.59 & Energy production and conversion \\
G & 205 & 8.37 & Carbohydrate transport and metabolism \\
E & 168 & 6.86 & Amino acid transport and metabolism \\
F & 77 & 3.14 & Nucleotide transport and metabolism \\
H & 83 & 3.39 & Coenzyme transport and metabolism \\
I & 54 & 2.20 & Lipid transport and metabolism \\
Q & 18 & 0.73 & Secondary metabolites biosynthesis, transport \\
& & & and catabolism \\
R & 0 & 0.00 & General function prediction only \\
S & 787 & 32.12 & Function unknown \\
Total & 2450 & 100.00 & \\
\hline & & &
\end{tabular}

homology (Additional file 1: Figure S2). OrthoANI values between strain CBA7123 and each of strains FORC 003, JP55, FORC 025, and JP838 were 96.2, 96.2, 96.3, and 95.8, respectively. Although the phylogenetic tree constructed based on these OrthoANI values did not obviously indicate that strain CBA7123 was clustered with the other C. perfringens strains (Fig. 1a), all of the OrthoANI values were over 95\%, which is the cut-off for species demarcation [17]. A POG comparison showed that the five strains shared 2392 POGs; however, 152 POGs were exclusive to strain CBA7123 (Fig. 2). Of the 152 POGs, some POGs encoded for antibiotic resistance and antiviral defense functions: two kinds of multidrug export MepA proteins, CRISPR-associated endonuclease Cas 1 and Cas2, $5^{\prime}$ to $3^{\prime}$ exodeoxyribonuclease. The dendrogram created based on the presence of POGs indicated that strain CBA7123 was clustered with strains JP55, FORC 003, and FORC 025 (Fig. 1b). These results revealed that strain CBA7123 is closely related to $C$. perfringens, but different from other $C$. perfringens strains.

\section{Pathogenesis, virulence factors, and antimicrobial resistance genes}

Strain CBA7123 was determined to be a human pathogen with 0.77 probability, using PathogenFinder 1.1. The 13 proteins except for conserved hypothetical proteins and hypothetical proteins were matched with pathogenic organisms belonging to class Clostridia (Table 3). The genome contained tetracycline-resistance genes: two $\operatorname{tet} A(\mathrm{P})$ genes, one each in the chromosome and plasmid; and one tet $B(\mathrm{P})$ gene in the plasmid. Strain FORC 003 genome also contained the same tetracycline-resistance genes, whereas strain JP838 genome only contained $\operatorname{tet} A(\mathrm{P})$. No antimicrobial resistance genes were detected in the other two strains. The key virulence factors of strain CBA7123 were predicted to be exo-alpha-sialidase (nanI), sialidase (nanH), collagenase (colA, kappa-toxin), perfringolysin $\mathrm{O}$ ( $p f o A$, theta-toxin), phospholipase $\mathrm{C}$ (plc, alpha-toxin), and alpha-clostripain (cloSI) with 96, 95, 98, 98 and $96 \%$ identity, respectively, in the chromosome and all of which were also present in the four reference strains. Sialidase hydrolyzes the $\alpha$-linkage of terminal sialic acid residues from mammalian cell surface to generate free sialic acid, which is important carbon and energy source for colonization and growth of bacterial pathogen in the intestines [22]. Perfringolysin $\mathrm{O}$ and phospholipase $\mathrm{C}$ are soluble toxin and phospholipid cleaving enzyme leading to host cell lysis, respectively, and these two enzymes have synergistic effects in Clostridium perfringens-mediated gas gangrene [23, 24]. Collagenase and alpha-clostripain are not major determinant of virulence in clostridial myonecrosis, but it could be assumed that the two proteins have effects on C. perfringens-mediated disease [25, 26]. Strain CBA7123 could be classified as toxinotype $\mathrm{A}$, based on the presence of the $\alpha$-toxin gene $(p l c)$. Identification of tetracyclineresistance genes and various other virulence factors may help future research into reducing the pathogenesis of $C$. perfringens in humans and contribute to faster treatment response. 
a
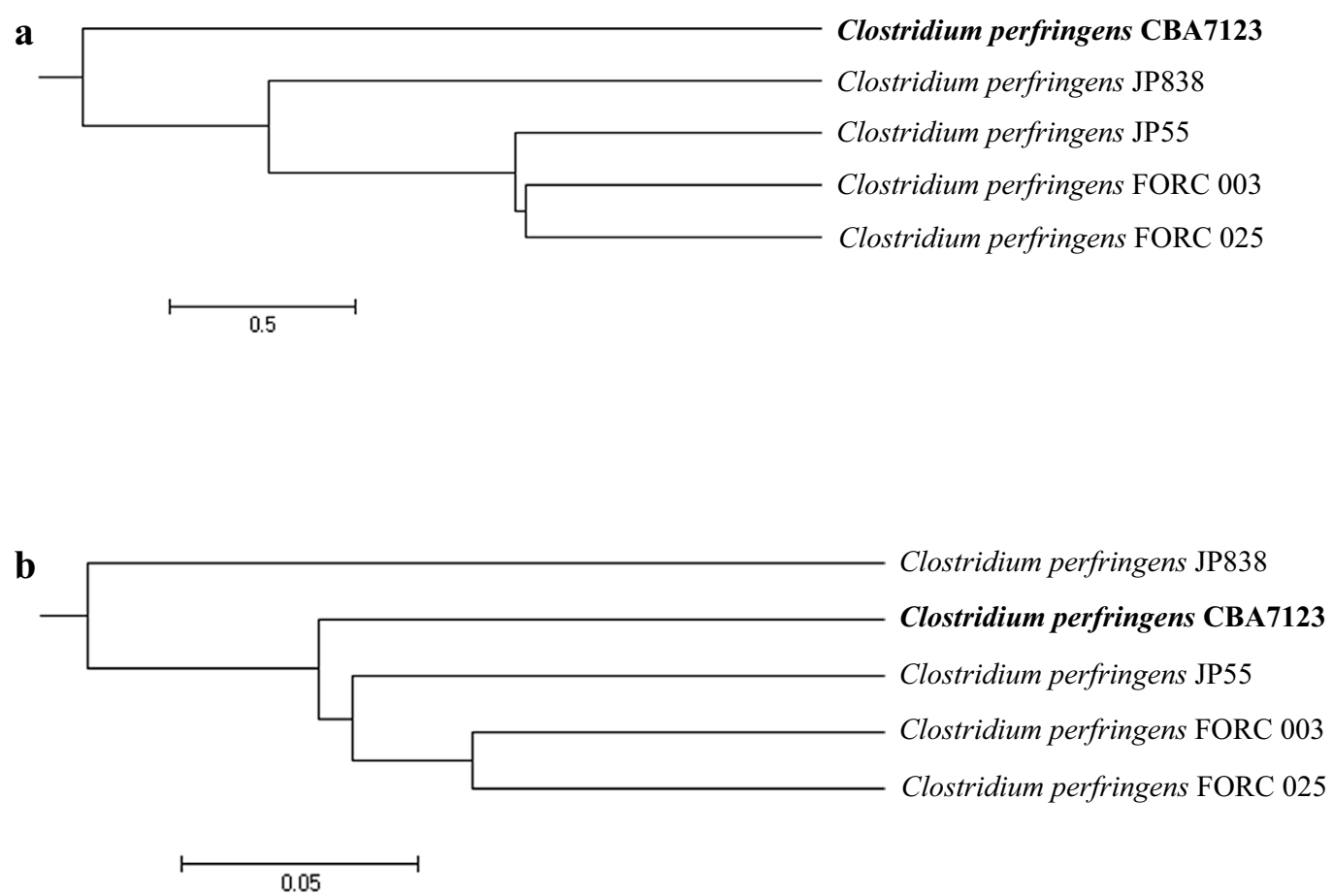

Fig. 1 Phylogenetic tree depicting the relationship between Clostridium perfringens CBA7123 and four reference strains: FORC 003, FORC 025, JP55, and JP838, constructed based on $\mathbf{a}$ OrthoANI values using the orthologous average nucleotide identity tool and $\mathbf{b}$ gene content (presence or absence) using Jaccard coefficients and UPGMA clustering

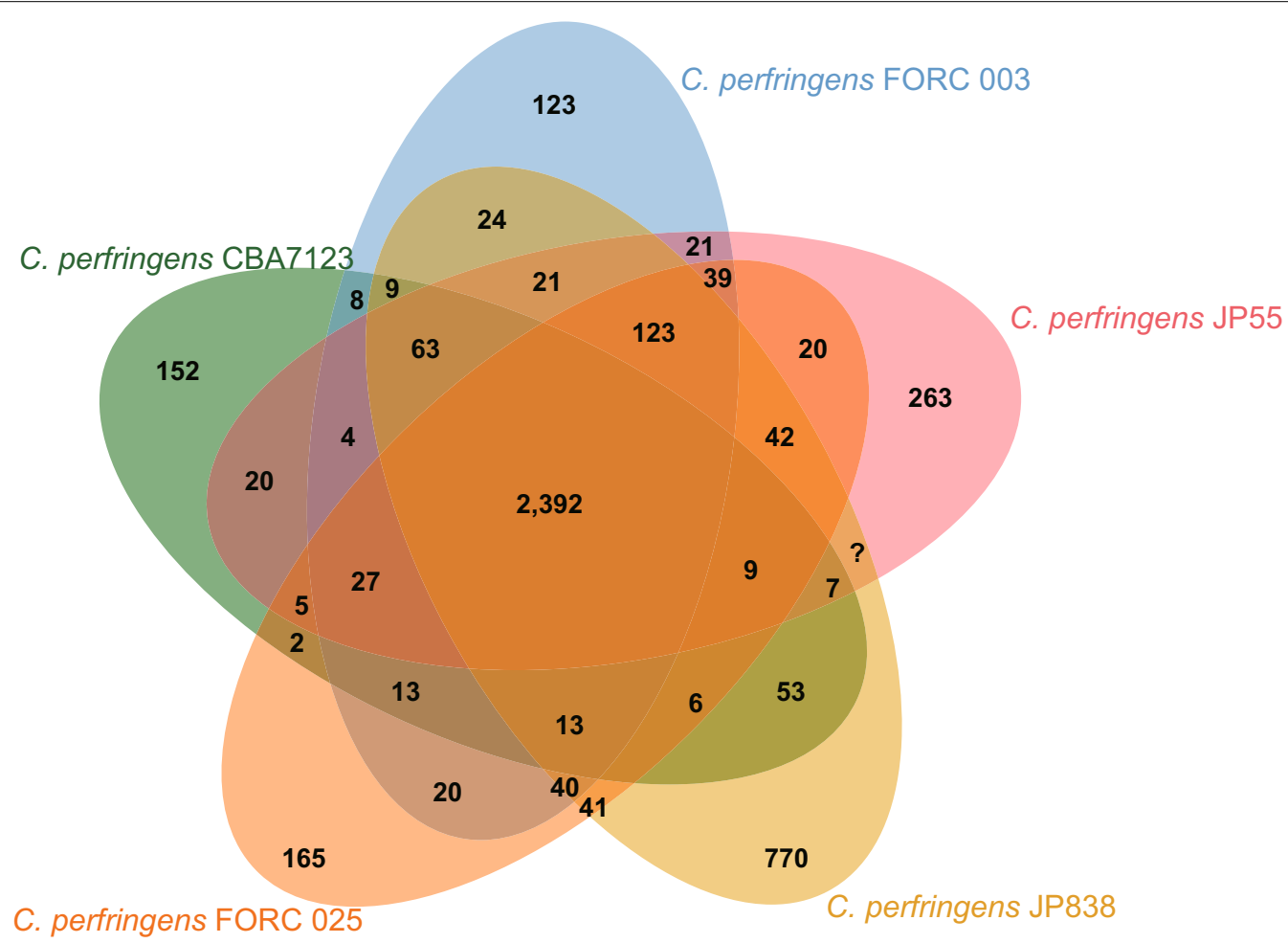

Fig. 2 Venn diagram based on POGs indicating the orthologous groups among five Clostridium perfringens strains. Number of Venn diagrams represents number of shared genes among the genomes of the five strains 
Table 3 Pathogenesis-related proteins matched to the genus Clostridium

\begin{tabular}{lll}
\hline Matched protein function & Matched organisms & Protein ID \\
\hline Acetyltransferase GNAT family & Clostridium perfringens ATCC 13124 & ABG84563 \\
Conserved hypothetical protein & Clostridium perfringens str. 13 & BAB81124 \\
Creatininase & Clostridium perfringens ATCC 13124 & ABS84729 \\
DedA family protein & Clostridium perfringens ATCC 13124 & ABG84228 \\
FemAB family protein & Clostridium perfringens SM101 & ABG87847 \\
Iron-sulfur cluster-binding protein & Clostridium perfringens SM101 & ABG85421 \\
Oxidoreductase, FAD-binding & Clostridium perfringens SM101 & ABG85949 \\
PTS system, mannose/fructose/sorbose family, IIC component & Clostridium perfringens ATCC 13124 & ABG82202 \\
Putative csfB protein & Clostridium perfringens SM101 & ABG87739 \\
Putative membrane protein & Clostridium perfringens ATCC 13124 & ABG83481 \\
Transcriptional regulator, PadR family & Clostridium perfringens ATCC 13124 & ABG82435 \\
Transcriptional regulator, PadR family & Clostridium perfringens SM101 & ABG85373 \\
TrkA domain protein & Clostridium perfringens ATCC 13124 & ABG84483 \\
\hline
\end{tabular}

\section{Future directions}

The genome information of strain CBA7123 can improve the understanding of $C$. perfringens, and the information of its antimicrobial resistance and virulence factors can contribute to the development of methods for preventing C. perfringens-related food poisoning. Future research studies should investigate the pathogenesis mechanism in detail as well as the specific roles of each virulence factor.

\section{Additional file}

Additional file 1: Figure S1. A photomicrograph of Clostridium perfringens strain CBA7123 using Variable Pressure Field Emission Scanning Electron Microscope (VP-FE-SEM). Figure S2. Comparison of genomic structure between Clostridium perfringens CBA7123 and strains FORC 003, FORC 025, JP55, and JP838, using a progressive alignment algorithm in Mauve. The locally collinear blocks with identical colors represent highly homologous regions. The genomes were figured based on scale of the genome of strain CBA7123.

\section{Abbreviations}

BLAST: Basic Local Alignment Search Tool; COGs: Clusters of Orthologous Groups; HGAP: Hierarchical Genome Assembly Process; KEGG: Kyoto Encyclopedia of Genes and Genomes; ORFs: open reading frames; POGs: pan-genome orthologous groups; RAST: Rapid Annotation using Subsystem Technology.

\section{Authors' contributions}

SWR and YDN designed and coordinated all the experiments. HSS performed the microbiology experiments. YBK, JYK, and SWR performed the bioinformatic analysis and wrote the manuscript. CL, JK, JK, JKR, and MSJ checked and edited the manuscript. All authors read and approved the final manuscript.

\footnotetext{
Author details

${ }^{1}$ Microbiology and Functionality Research Group, World Institute of Kimchi, Gwangju 61755, Republic of Korea. ${ }^{2}$ Biological Disaster Analysis Group, Korea Basic Science Institute, Daejeon 34133, Republic of Korea. ${ }^{3}$ Gut Microbiome Research Group, Korea Food Research Institute, Seongnam 13539, Republic of Korea. ${ }^{4}$ University of Science and Technology, Daejeon 34113, Republic of Korea. ${ }^{5}$ Department of Food Science and Engineering, Ewha Womans
}

University, Seoul 03760, Republic of Korea. ${ }^{6}$ Chuncheon Center, Korea Basic Science Institute, Gangneung, Gangwon-do 24341, Republic of Korea.

\section{Acknowledgements}

Not applicable.

\section{Competing interests}

The authors declare that they have no competing interests.

\section{Availability of data and materials}

This complete genome project has been deposited at DDBJ under the accession numbers AP017630-AP017631.

\section{Ethics approval and consent to participate}

The study protocol was approved by the institutional review board of the Theragen ETEX Bio Institute (700062-20160804-JR-005-02).

\section{Funding}

This research was supported by the Korea Food Research Institute (E017060201), the World Institute of Kimchi funded by the Ministry of Science, ICT and Future Planning, Republic of Korea (KE1702-2), the Center for Analytical Research of Disaster Science of the Korea Basic Science Institute (C37703), and the Basic Science Research Program through the National Research Foundation of Korea (NRF) funded by the Ministry of Education, Science, and Technology (2015R1D1A1A09061039).

\section{Publisher's Note}

Springer Nature remains neutral with regard to jurisdictional claims in published maps and institutional affiliations.

Received: 13 March 2017 Accepted: 25 May 2017

Published online: 02 June 2017

\section{References}

1. Minton NP, Ehsaan M, Humphreys CM, Little GT, Baker J, Henstra AM, et al. A roadmap for gene system development in Clostridium. Anaerobe. 2016;41:104-12.

2. Shimizu T, Ohtani K, Hirakawa H, Ohshima K, Yamashita A, Shiba T, et al. Complete genome sequence of Clostridium perfringens, an anaerobic flesh-eater. Proc Natl Acad Sci USA. 2002;99:996-1001.

3. Wong YM, Juan JC, Gan HM, Austin CM. Draft genome sequence of Clostridium perfringens strain $\mathrm{JJC}$, a highly efficient hydrogen producer isolated from landfill leachate sludge. Genome Announc. 2014;2:e00064. 
4. Johansson A, Aspan A, Bagge E, Baverud V, Engstrom BE, Johansson KE. Genetic diversity of Clostridium perfringens type $A$ isolates from animals, food poisoning outbreaks and sludge. BMC Microbiol. 2006;6:47.

5. Gross TP, Kamara LB, Hatheway CL, Powers P, Libonati JP, Harmon SM, Israel E. Clostridium perfringens food poisoning: use of serotyping in an outbreak setting. J Clin Microbiol. 1989;27:660-3.

6. Hassan KA, Elbourne LD, Tetu SG, Melville SB, Rood JI, Paulsen IT. Genomic analyses of Clostridium perfringens isolates from five toxinotypes. Res Microbiol. 2015;166:255-63.

7. Petit L, Gibert M, Popoff MR. Clostridium perfringens: toxinotype and genotype. Trends Microbiol. 1999;7:104-10.

8. Rood JI, Cole ST. Molecular genetics and pathogenesis of Clostridium perfringens. Microbiol Rev. 1991;55:621-48.

9. Brynestad S, Granum PE. Clostridium perfringens and foodborne infections. Int J Food Microbiol. 2002;74:195-202.

10. Hashiba M, Tomino A, Takenaka N, Hattori T, Kano H, Tsuda M, Takeyama N. Clostridium Perfringens Infection in a Febrile Patient with Severe Hemolytic Anemia. Am J Case Rep. 2016;17:219-23.

11. Kim JY, Song HS, Kim YB, Kwon J, Choi JS, Cho YJ, et al. Genome sequence of a commensal bacterium, Enterococcus faecalis CBA7120, isolated from a Korean fecal sample. Gut Pathog. 2016;8:62.

12. Chin CS, Alexander DH, Marks P, Klammer AA, Drake J, Heiner C, et al. Nonhybrid, finished microbial genome assemblies from long-read SMRT sequencing data. Nat Methods. 2013;10:563-9.

13. Cosentino S, Voldby Larsen M, Moller Aarestrup F, Lund O. PathogenFinder-distinguishing friend from foe using bacterial whole genome sequence data. PLoS ONE. 2013;8:e77302.

14. Zankari E, Hasman H, Cosentino S, Vestergaard M, Rasmussen S, Lund O, et al. Identification of acquired antimicrobial resistance genes. J Antimicrob Chemother. 2012;67:2640-4.

15. Chen L, Yang J, Yu J, Yao Z, Sun L, Shen Y, Jin Q. VFDB: a reference database for bacterial virulence factors. Nucleic Acids Res. 2005;33:D325-8.

16. Darling AC, Mau B, Blattner FR, Perna NT. Mauve: multiple alignment of conserved genomic sequence with rearrangements. Genome Res. 2004;14:1394-403.
17. Lee I, Kim YO, Park SC, Chun J. OrthoANI: an improved algorithm and software for calculating average nucleotide identity. Int J Syst Evol Microbiol. 2015:66:1100-3.

18. Ward N, Moreno-Hagelsieb G. Quickly finding orthologs as reciprocal best hits with BLAT, LAST, and UBLAST: how much do we miss? PLOS ONE. 2014;9:e101850.

19. Yoon SH, Ha SM, Kwon S, Lim J, Kim Y, Seo H, Chun J. Introducing EzBioCloud: a taxonomically united database of $16 \mathrm{~S}$ rRNA and whole genome assemblies. Int J Syst Evolut Microbiol. 2016. (In press).

20. Bardou P, Mariette J, Escudie F, Djemiel C, Klopp C. jvenn: an interactive Venn diagram viewer. BMC Bioinform. 2014:15:293

21. Min UG, Kim SJ, Hong H, Kim SG, Gwak JH, Jung MY, et al. Calculibacillus koreensis gen. nov., sp. nov., an anaerobic Fe(III)-reducing bacterium isolated from sediment of mine tailings. J Microbiol. 2016;54:413-9.

22. Li J, Uzal FA, McClane BA. Clostridium perfringens Sialidases: potential contributors to intestinal pathogenesis and therapeutic targets. Toxins. 2016;8:341.

23. Awad MM, Ellemor DM, Boyd RL, Emmins JJ, Rood Jl. Synergistic effects of alpha-toxin and perfringolysin $\mathrm{O}$ in Clostridium perfringens-mediated gas gangrene. Infect Immun. 2001;69:7904-10

24. Dang TX, Hotze EM, Rouiller I, Tweten RK, Wilson-Kubalek EM. Prepore to pore transition of a cholesterol-dependent cytolysin visualized by electron microscopy. J Struct Biol. 2005;150:100-8.

25. Awad MM, Ellemor DM, Bryant AE, Matsushita O, Boyd RL, Stevens DL, et al. Construction and virulence testing of a collagenase mutant of Clostridium perfringens. Microb Pathog. 2000;28:107-17.

26. Chakravorty A, Awad MM, Hiscox TJ, Cheung JK, Carter GP, Choo JM, et al. The cysteine protease alpha-clostripain is not essential for the pathogenesis of Clostridium perfringens-mediated myonecrosis. PLOS ONE. $2011: 6: e 22762$

\section{Submit your next manuscript to BioMed Central and we will help you at every step:}

- We accept pre-submission inquiries

- Our selector tool helps you to find the most relevant journal

- We provide round the clock customer support

- Convenient online submission

- Thorough peer review

- Inclusion in PubMed and all major indexing services

- Maximum visibility for your research

Submit your manuscript at www.biomedcentral.com/submit
O Biomed Central 